\title{
Three-element Compound Drive Target Delivery to Improve the Recovery Technology
}

\author{
Yu Zhang \\ No.1 oil Filed Geological Team, No.3 Oil Production plant, Daqing Oilfield Co., Ltd., Daqing \\ 163000, China
}

\begin{abstract}
A lot of researches have been carried out on the loss of three-element compound system at home and abroad, it is thought that permeability of three-element compound system in oil layer are mainly physical factors, chemical adsorption, mechanical trapping and chromatograph and so on in the research. Under such a background, finding three-element compound drive target delivery to improve the recovery technology can better promote technological reform and development. This paper mainly discusses how to grasp the dynamic characteristics of three-element compound drive target delivery to improve the recovery technology, adjust the parameters and improve recovery ratio.
\end{abstract}

Keywords: three-element compound drive; target delivery; recovery ratio; technology; improvement.

\section{Introduction}

The three-element compound drive is typical representatives of the tertiary oil recovery technology, which can greatly improve the oil recovery ratio; however, the loss of the three-element compound system appears in the formation, which is also an important bottleneck to restrict the development of this technology, especially in the vicinity of the injection well, it is more sufficient to flush near the well after water flooding, and there is more water saturation in this place, at this time, if the threeelement compound system is injected, it will bring more serious loss, finally make the performance of the ternary composite system seriously affected.

\section{Dynamic Characteristics of Three-Element Compound Drive Target Delivery}

In a large number of experiments, people found that after a period of three-element compound drive, as the three-element compound system moved and shifted toward the depth of the model, the overall moisture content began to decline under various injection modes. Under the conventional injection mode, the three-element compound system will transfer along the previous permeation channel, but under the target injection mode, the three-element compound system will follow the channel in the direction and quickly assemble in the remaining oil enrichment region, under such circumstances, the overall moisture content of the targeted injection mode decline faster, and the water content reduction curves in the case of such two target injection methods are basically identical. For the conventional three-element compound flooding moisture, the lowest state can be reduced to about 35\%, and the maintenance time of lowest moisture content is also relatively short. When the water content reaches the lowest point, under the conventional injection method, the water content will rise rapidly, and it can reach 98\% state quickly and slowly reach a stable state. For the target injection method, the increase of water content is relatively slow and stable, due to the right angle target injection method, the whole area affected is relatively large, and the efficiency affected is relatively high, which will replace more residual oil, so in the subsequent water flooding phase, the rise rate and extent of water content will be smaller than the target angle injection method. After contrast various methods, people have also found that the method of right-angled target injection through experiments, which can be more effective in washing and controlling oil.

In the three-element compound drive stage, in general, the rate and the extent of increase of recovery degree of the targeted injection method are more desirable than the conventional injection method. In the subsequent water drive stage, the change in the recovery degree of the three-element compound drive under normal conditions gradually reaches a steady state, and the final recovery degree generally reaches about 53\%. The output degree of target displacement will increase to a 
certain extent, and then slowly reach a stable state, and final recovery ratio will reach about $60 \%$. For right-angle target displacement recovery ratio, compared to opposite angles target displacement, the higher level is about $5.7 \%$ to $6 \%$, and the final recovery ratio will reach $70 \%$ to $71 \%$ level. For the target delivery method, it will have higher spread efficiency, so the overall oil displacement effect is ideal, and the way of targeted injection will be more ideal.

\section{Optimize the Parameters of Target Delivery}

In order to better improve the recovery ratio of three-element compound drive target delivery, some scientific measures and means should be taken to better improve the overall recovery ratio and the final effect. Optimizing the target delivery parameters is a very important link, CMG numerical simulation software can be used to simulate the radial three-element compound drive of the flat model, in this way, and we can better study the length of different target channels, injection amount of threeelement compound system and various changes in displacement effects, in this way, find the best target displacement solution. Under normal circumstances, the basic parameters of the numerical model are consistent with the actual physical model; therefore, this relationship can be used as a guide to optimize parameters.

\subsection{Length Optimization of Target Channel}

In order to better find the optimal target channel length, it should ensure entire operation and physical simulation experiment process are consistent when simulating displacement process. First, water drive is carried out, when the water content reaches about $98 \%$, the targeted channel can be opened, and 0.3-times pore volume three-element compound system is injected into the channel, and then water drive operation is performed until the overall water content reaches $98 \%$, the process of displacement at this time can be considered to be over.

When water content of simulation calculation water drive reaches $98 \%$, the recovery ratio is generally about 35\%. After the water drive is over, we can first ensure that the other conditions remain the same, and then set the channel length to a different length state. When setting the length, we need to ensure a reasonable length gradient between each other. In a large number of experiments, it can be found that when the length of target channel is zero, this state is a relatively conventional threeelement compound drive, compared with water drive; the recovery ratio in this case is about $23 \%$. When the length of the angle target channel increases from $4 \mathrm{~cm}$ to $35 \mathrm{~cm}$, the recovery ratio corresponding to the length is also in a state of rising first and then decreasing. In general, when the length of the channel is equal to $10 \mathrm{~cm}$, a maximum value is reached, which is generally about $36 \%$. Under the right angle target injection method, the trend of the rise curve of the recovery ratio is consistent with the way of diagonal target, but from an objective angle, the overall recovery ratio is generally better than diagonal target. When the length of the target channel is $10 \mathrm{~cm}$, the maximum value will generally reach about $47 \%$. From the overall simulation results, it can be found that the optimal length of the target channel is generally about $10 \mathrm{~cm}$, and the ratio among the well spacing is about $14.14 \%$. 


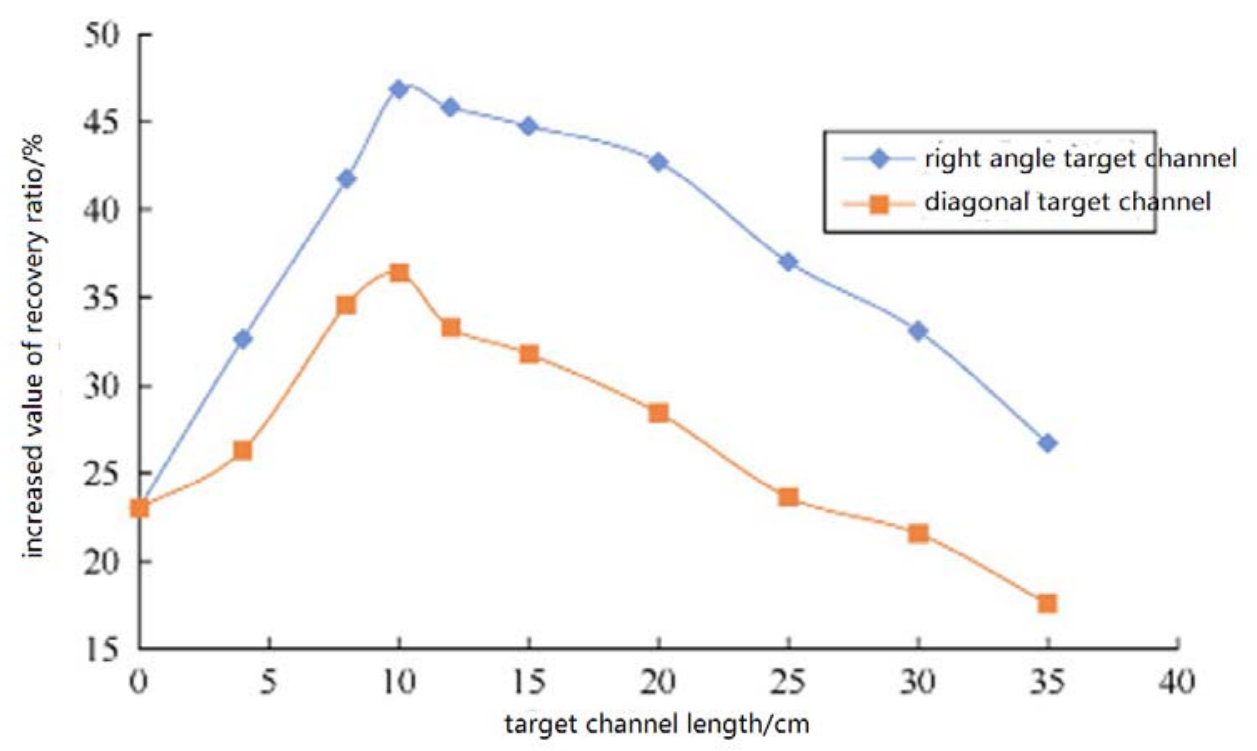

Fig.1 Simulate the water content of water flooding

From the relationship diagram between the length and recovery ratio increase of the target channel, it can be found that as the target channel becomes longer, three-element compound reduces some unnecessary losses, thus causing recovery ratio get dramatic increase. As the channel length continues to increase, in general, the increase in recovery ratio will only decrease under normal conditions. After analyzing the cause, it can be found that near-well zone of the injection well; this place is generally fully flushed during the process of water drive. In addition, the remaining oil is also relatively small in this place, and generally gathers in the middle and rear parts. Under normal circumstances, the target channel with proper length can effectively avoid the area where the remaining oil is relatively small, and finally the washing effect of three-element compound system can be fully exerted. If the degree of the target channel is too long, then some remaining oil distribution area will be skipped, under such a situation, the spread area will decrease, and the increase rate of the generation rate will also decrease. .

\subsection{Optimization of Target Channel Position}

Through the previous series of operations, combined with some specific experimental design, we can help people choose the best target channel design scheme. For the results of the position optimization simulation of the target channel, specific data can be read from the following table:

Table 1. parameter table for optimization simulation of target channel

\begin{tabular}{|c|c|c|c|c|}
\hline $\begin{array}{c}\text { simulation } \\
\text { scheme }\end{array}$ & $\begin{array}{c}\text { target channel } \\
\text { method }\end{array}$ & $\begin{array}{c}\text { target channel } \\
\text { method } / \mathrm{cm}\end{array}$ & $\begin{array}{c}\text { injection pore volume } \\
\text { multiple }\end{array}$ & $\begin{array}{c}\text { increased value of } \\
\text { recovery ratio }\end{array}$ \\
\hline 1 & no & 0 & 0.4 & $26.12 \%$ \\
\hline 2 & diagonal target & 10 & 0.4 & $40.29 \%$ \\
\hline 3 & right angle target & 10 & 0.4 & $48.90 \%$ \\
\hline 4 & compound target & 10 & 0.4 & $43.89 \%$ \\
\hline
\end{tabular}

Compared with the way of water drive, the no-target channel method is the conventional threeelement compound system injection method, it increase by $26.12 \%$ in recovery ratio. It can be found from these data that the opposite angles, right angle and compound target are $10 \mathrm{~cm}$, inject threeelement compound system with 0.4 times pore volume, compared with the way of water drive, the overall recovery ratio increase by $40.29 \%, 48.90 \%$ and $43.89 \%$, right angle target increase highest recovery ratio among these three methods, compared to diagonal target, right angle target will have 
higher sweep efficiency, therefore, it will be more obvious in the improvement effect of the recovery ratio. Although compound target method has more target channels and larger spread area, under the condition of a fixed amount of three-element compound system, the amount of compound system in each target channel will decrease, so the compound system will be diluted and lost faster, and the corresponding displacement efficiency will also be reduced. When the total amount of three-element compound system is certain, it is not as people imagine, the degree of recovery ratio increases as the target channels increase, in short, compound target three-element compound is not as good at improving the recovery ratio as right angle target. Combined with the results of these experiments and analyses, right angle target is the best choice in target design.

\section{Conclusion}

In summary, the delivery technology of three-element compound target can effectively integrate the high-pressure water jet drill radial horizontal well technology and the three-element compound drive phase, under such circumstances, the three-element compound system can be directly transported to the remaining oil-rich areas by people, this way can effectively avoid the loss in the near-well zone of the water injection well, which can significantly increase the sweep efficiency, and ultimately improve the overall oil displacement effect, at the same time, the recovery ratio of crude oil can be greatly improved. With the continuous advancement of research work, delivery technology of three-element compound target will play an active role in the improvement of recovery ratio; effectively solve the high loss problem of chemical agents in the near-well zone of ternary composite flooding. It can compensate for the high cost of three-element compound drive to a certain extent, solve the defects of limited application, and has a broad application prospect in the development of the market.

\section{References}

[1]. Hou Jirui, Wu Fan, Ma Yunfei. Study on EOR by ASP of Targeted Delivery [J]. Oilfield Chemistry, 2017(03):524-527.

[2]. Wei Lixin, Pang Renshan, Zhuge Xianglong. Experimental study on deposition regulation in oil gathering pipeline by ASP flooding Process [J]. Xinjiang Petroleum Geology, 2012(06):741-743.

[3]. Yang Haiying. CO2 flooding ground engineering [J]. Petrochemical Industry Technology, 2016(08):145.

[4]. Cui Zheng, Zhu Yongdong. Research progress on enhanced oil recovery technology of chemical drive in heavy oil fields [J]. Inner Mongolia Petrochemical Industry, 2013 (04): 153-156.

[5]. Liu Gang, Hou Jirui, Li Qiuyan. Chemicals loss and effective distance of ASP flooding in secondclass oil layers [J]. Journal of China University of Petroleum: Natural Science Edition, 2015(06): 171-177.

[6]. Leng Jun, Pan Yi, Li Dongsheng. Application of Chemical Flooding Technology [J].Contemporary Chemical Industry, 2014 (08): 1495-1497. 